\title{
Knowledge, Attitudes and Practices About Contraception in Patients who had Undergone A Caesarian Section in N'Djamena Mother and Child University Hospital
}

\author{
Gabkika Bray Madoué ${ }^{1 *}$, Foumsou Lhagadang ${ }^{1}$, Saleh Abdelsalam² and Hinsoubé Guelo ${ }^{2}$ \\ ${ }^{1} N^{\prime}$ Djamena Mother and Child University Hospital, Chad \\ ${ }^{2} N^{\prime}$ Djamena faculty of health human sciences, Chad
}

*Corresponding author: Gabkika Bray Madoué, Department of genecology and obstetrics, N`Djamena Mother and Child University Hospital, Chad.

\section{Abstract}

Background: Postpartum contraception is the set of methods designed to prevent pregnancy after a caesarean section [1]. It can be instituted during the caesarean section or in the post-partum period.

The aim of this study is to evaluate the knowledge and practice of contraception in patients who have undergone a caesarean section at N'Djamena Mother and Child University Hospital.

Patients and method: This were a 3-month cross-sectional and descriptive study from September 1st, 2020 - November 30th, 2020 on knowledge, attitudes and practices about contraception in caesarian section patients.

Results: The 20-24 age group was the most represented with 42\%.Patientts resided in N'Djamena in 60\%.Married women were the most represented with $93.3 \%$. Patients had average knowledge about contraception in $77.3 \%$. Injectable contraceptives were the commonly known of the modern contraceptive methods with $80.7 \%$. Periodic abstinence was the best-known natural method with $61.3 \%$. Implants were the most chosen contraceptive method with $48 \%$. The proposal to use the contraceptive method was made in $79,3 \%$ of cases by health workers. The most well-known advantages of contraceptives by clients were birth spacing with $82.7 \%$. Infertility was the contraceptive method disadvantage most reported by clients with $54 \%$.

Conclusion: The prescription of contraceptive methods in patients who have undergone a caesarean section is part of our common practice. Injectable contraception remains the commonly known hormonal contraception. The desire to avoid pregnancy is the benefit most noted by patients.

Keywords: Contraception; Caesarean section; NMCUH

\section{Introduction}

Postpartum contraception is the set of methods designed to prevent pregnancy after a caesarean section [1]. It can be instituted during the caesarean section or in the post-partum period. There is a disparity in the rate of use of contraceptive methods around the world. The rate of modern contraceptive use is $55 \%$ worldwide, reaching $22 \%$ in Africa with a rate less than $10 \%$ in its central part $[1,2]$. These disparities in contraceptive use rates also exist between urban and rural areas. For example, high rates can be observed in

urban areas compared to rural areas. In Chad, according to data from the 2015 mixed survey, the prevalence of modern contraception is less than 5\% for all women of childbearing age [3]. Foumsou L, et al [4] in a hospital series at N'Djamena Mother and Child University hospital in 2016 reported that the rate of contraceptive use in the post-partum period is $15.9 \%$. However, several factors such as social and cultural factors are involved in the use of contraceptives. Few data exist on contraception after Caesarean section in our context. 
The aim of this study is to evaluate the knowledge and practice of contraception in patients who have undergone a caesarean section at N'Djamena Mother and Child University Hospital.

\section{Patients and Methods}

This was a three (3) month cross-sectional and descriptive study from September 1, 2020 - November 30, 2020 on knowledge, attitudes and practices about contraception in caesarian section patients.

Patients were interviewed. According to the answers given during the interview, the knowledge of the patients is classified as follows:

Table 1: Age distribution.

\begin{tabular}{|c|c|c|}
\hline Age (year) & $\mathbf{n}$ & \% \\
\hline$<20$ & 18 & 12 \\
\hline $20-24$ & 63 & $21 / 3$ \\
\hline $25-29$ & 32 & 18.7 \\
\hline $30-34$ & 28 & 4 \\
\hline $35-39$ & 6 & 2 \\
\hline$\geq 40$ & 3 & 100 \\
\hline Total & 150 & \\
\hline
\end{tabular}

The $20-24$ age group was the most represented with $42 \%$.

The average age was $25.31 \pm 5.51$ years with extremes of 17 and 45 years.

Marital status: Married women were the most represented with $93.3 \%$ of the cases ( $\mathrm{n}=140)$, among the remaining we noted: 3 living with a partner (2\%), 6 divorced $(4 \%)$ and one single $(0.7 \%)$.

Level of education: Patients with primary education were most represented with $36.7 \%(n=55)$, followed by those with no schooling $(28.7 \%, n=43)$ and secondary education $(20.7 \%, n=31)$.

Parity: Parity was dominated by paucipares $44.7 \%(\mathrm{n}=67)$, followed by multiparous $(34 \%, \mathrm{n}=51)$ and primiparous $(21.3 \%$, $\mathrm{n}=32)$.

Table 2: knowledge of modern methods of contraception.

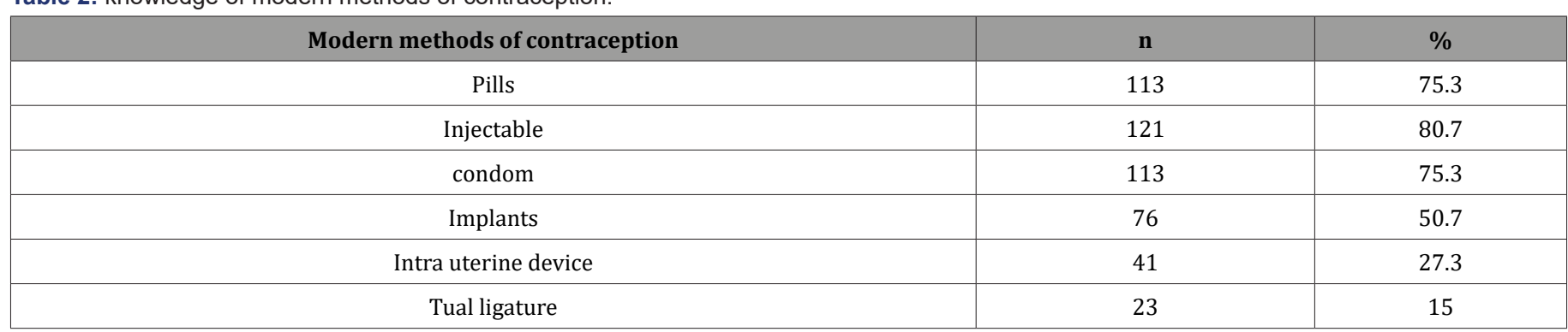

Injectable contraceptives were the commonly known of the modern contraceptive methods with $80.7 \%$.

Table 3: Distribution of users according to the chosen method.

\begin{tabular}{|c|c|c|}
\hline Chosen method & $\mathbf{n}$ \\
\hline Pills & 13 & \\
\hline Injection & 5.7 & 58 \\
\hline Implant & 38.7 & 72 \\
\hline
\end{tabular}




\begin{tabular}{|c|c|c|}
\hline Intra uterine device & 7 & 4.7 \\
\hline Total & 150 & 100 \\
\hline
\end{tabular}

Implants were the most chosen contraceptive method with $48 \%$ of cases.

Table 4: Proposal to use the contraceptive method.

\begin{tabular}{|c|c|c|}
\hline Proposal to use contraception & n & \% \\
\hline Partner & 8 & 15.3 \\
\hline Patient herself & 23 & 79.3 \\
\hline Health worker & 119 & 100 \\
\hline Total & 150 & \\
\hline
\end{tabular}

The proposal to use the contraceptive method was made in $79,3 \%$ of cases by health workers.

\section{Advantages of the contraceptive method}

The most well-known advantages of contraceptives by clients were birth spacing with $82.7 \%$. This was followed by avoiding unwanted pregnancies $(66 \%, \mathrm{n}=99)$ and avoiding STI/HIV (38\%, $\mathrm{n}=57)$.

\section{Disadvantages of contraceptive methods}

Infertility was the contraceptive method disadvantage most reported by clients with $54 \%$.soot by weight gain $(48.7 \%, n=73)$, and cycle disorder $(48 \%, \mathrm{n}=72)$.

\section{Discussion}

According to Lansac [5], the need for contraception is high among sexually active young people. This assertion is corroborated in this series with an average age of contraceptive users of 25.31 5.51 years. The age group between 20 and 24 years old was the most represented with $42 \%$. This result is close to that of Foumsou, et al [4] in N'Djamena in 2016 who reported an average age of 24.1 years and a high proportion of the 21-25 age group with $32.1 \%$.

National data show a high rate of contraceptive use in urban areas compared to rural areas. We noted that $60 \%$ of clients resided in the city of N'Djamena against 40\% outside N'Djamena (rural). This result is higher than that of Rahariniaina, et al. [6] in Ramainandro in Madagascar in 2015 who noted that 34\% of clients lived in urban areas $34 \%$ versus $20 \%$ in rural areas.

Regarding marital status, married women represented $93 \%$ of the cases. The same observation was made by Foumsou, et al. [7] in N'Djamena Chad in 2015 who point out that $88.4 \%$ of patients were married. These results could be explained by cultural factors. Indeed, the occurrence of pregnancy outside marriage is not tolerated by many tribes in Chad. The occurrence of pregnancy outside of marriage is experienced as a disgraceful factor for families.

According to the literature $[7,8]$, birth control becomes a requirement or an imperative if the number of children increases. This rhymes with a high proportion of multiparous women among clients requesting contraceptives. This series contrasts this observation with a higher proportion of paucipara. This could be explained by the haunting of iterative caesarean sections forcing patients to limit maternity.

According to recent data, information on contraceptive methods is provided through the usual means of communication [9]. Patients' level of knowledge about different contraceptive methods may be related to their level of education and general culture. Thus, clients' knowledge of contraceptive methods may be scattered. We noted that patients with average knowledge of contraceptive methods accounted for $77.3 \%$. This rate is lower than that reported by Byamungu, et al. [10] in Bukavu in DRC in 2015 and Leye, et al. [8] reported a good knowledge of contraceptive methods of $79.3 \%$ and $81 \%$ respectively. The rate obtained in this series is thought to be linked to the high proportion no schooled and primary level patients (65.4\%). In fact, schooling is a means of increasing knowledge globally and general culture more specifically.

Injectable contraceptives were most commonly known by clients with $80.7 \%$ Foumsou, et al. [4] in a previous study found a similar rate with $81 \%$ of clients knowing about injectable contraceptives. The same is true for Rahariniaina, et al. [6] who found a rate of $77.8 \%$ of clients knowing about injectable contraceptives. The availability of the product in many health care settings, especially the easier mode of use and the long duration of action may explain these results.

In the absence of hormonal contraception, the natural method can be used to prevent pregnancy. There are many such methods and knowledge about them is often based on their effectiveness. We noted that $61.3 \%$ of clients knew about periodic abstinence. This result is higher than Foumsou, et al. [7] found that users knew about periodic abstinence as a contraceptive method in $59.4 \%$.

In order to avoid pregnancy in clients with uterine scarring, a long-acting contraceptive method is needed. This will help to avoid the mistakes associated with forgetting to take the pill $[4,7]$. We reported that $48 \%$ of the clients had opted for the implant. This result is close to that of Gennai in Monpellier, France in 2012 [11] who reported an implant use rate of $51.9 \%$.

In general, the antenatal and postnatal consultations are also devoted to educating patients to improve their condition by offering them contraception, which appears to be a safe method to avoid pregnancy [12], thus the staff can encourage a couple to 
use contraception. This remains true in this series, with $79.3 \%$ of clients reporting being counselled by health workers on the use of contraception. This result differs from that of Coulibaly, et al. [13] in Cote d'Ivoire in 2019 , who point out that $48 \%$ had been counselled by their spouses. This result can be explained by the overall decision in the couple.

Birth spacing remains the most frequently cited advantage for the use of contraceptive methods in certain African series [6-8]. This series corroborates those of these authors with $82.7 \%$ who cited birth spacing as the advantage of using contraception.

One of the factors that can hinder the use of contraceptives is bleeding [5]. However, other embarrassing effects, such as non-permanent sterility, are often cited as reasons for limiting contraceptive use. More than half of the clients (54\%) cited infertility as a disadvantage of contraceptive methods. This result is higher than Barry [14] in Bamako in 2015, who found that 27\% of users considered infertility to be the disadvantage of contraceptive methods.

\section{Conclusion}

The prescription of contraceptive methods in patients who have undergone a caesarean section is part of the common practice at N'Djamena Mother and child Hospital. The demographic characteristics of the clients are young, married and mostly urban. The clients' level of knowledge and their practice are diverse. Injectable contraception remains the commonly known hormonal contraception. Among the natural contraceptive methods, Interrupted coitus is the best known. The decision to use contraception is facilitated by the health staff. The implant is the most commonly used method of contraception. The desire to avoid pregnancy is the benefit most noted by patients. The most reported disadvantage is infertility.

Through this study we can say that there is still a need to raise awareness among pregnant women in order to further boost the practice of contraception in patients who have undergone a caesarean section.

\section{Acknowledgement}

None.

\section{Conflict of Interest}

Authors declare no conflict of interest.

\section{References}

1. Gronier H, Robing, Dewaily D (2011) Contraception. Practitioner's view. 61: 849-857.

2. Sépou A, Enza J, Nali NM (2000) Difficulties related to the expansion of Contraception in urban and semi-urban areas of the Central African Republic. Med Afri Noire 47(2) :73-78

3. (2015) National Institute of Statistics for Economic and Demographic Studies (INSEED). Chad Demographic and Health Survey III(EDST3).

4. Foumsou L, Gabkika BM (2018) Post partum hormonal contraception at the Mother and Child Hospital of N'Djamena. Annales de l'Université de N’Djamena Série C 10: 97-108.

5. Lansac J, Body G (2012) Gynaecology. Paris: Masson.

6. Rahariniaina M (2015) Knowledge, attitudes, practices and modern contraceptive prevalence. Ramainandro: University of Tananarive.

7. Foumsou L, Dangar GD, Djimasra TO (2015) Problem of Family Planning in the Hospitals of the city of N'Djamena. Med Afr Noire 62(4): 230-236.

8. Leye MM, Niang K, Seck I, Camara PI, Faye A, et al. (2012) Knowledge, attitudes and practices of modern contraception in the health district of Bambeye, Senegal. Med Afr Noire 59(9): 430-436.

9. Gabkika BM, Foumsou L, Adamou MA, Doune N, Tchoubou BM

10. (2016) Annales de l’Université de Bangui Série D 2( $\left.\mathrm{N}^{\circ} 001\right):$ 27-30.

11. Byamungu N, Kabinda J, Mvula M (2015) Knowledge and attitudes of adolescents in the Discrit Sanitaire of Bukavu (DRC) about contraception. Rev med Gd lacs 4(1): 40-46.

12. Gennai J (2012) Contraception et précarité : enquête auprès de femmes consultant en centre de protection maternelle et infantile. Montpellier :'Université de Montpellier, France.

13. Gabkika BM, Foumsou L, Saibana G, Abdelsalam S (2019) Contraception Method Following Spontaneous Abortion in N'djamena Mother and Child Hospital. South Asian Res J Nurs Health Care 1(2): 69-71.

14. Coulibaly M (2019) Perceptions and practices of contraception in an urban co-community in the city of Abidjan. Abidjan: Université Félix Houphouet Boigny.

15. Barry Sidibé (2015) Studies on the knowledge, attitudes and practices of resident students at the FAST Campus in Bamako in the area of family planning. University of Bamako. 